\title{
Three Dimensions of Labor: Cognitive Labor Differentiated from Emotional and Physical Labor
}

\author{
Sih Lee ${ }^{1}$, Junghye Jeong ${ }^{2}$, Yang Lee ${ }^{3}$ \\ ${ }^{1}$ Department of Economics, State University of New York, Albany, NY, USA \\ ${ }^{2}$ Department of Mathematics, State University of New York, Albany, NY, USA \\ ${ }^{3}$ Department of Psychology, Gyeongsang National University, Jinju, South Korea
}

Email address:

lee.sih@gmail.com (S. Lee), jeongpsy81@gmail.com (J. Jeong), yangleepsy@gmail.com (Y. Lee)

\section{To cite this article:}

Sih Lee, Junghye Jeong, Yang Lee. Three Dimensions of Labor: Cognitive Labor Differentiated from Emotional and Physical Labor. Journal of Human Resource Management. Vol. 5, No. 4, 2017, pp. 57-62. doi: 10.11648/j.jhrm.20170504.11

Received: August 2, 2017; Accepted: August 15, 2017; Published: September 21, 2017

\begin{abstract}
This study attempted to break limitations of traditional dimensions of labor, as pronounced in the physical and emotional labor, and propose a third dimension of cognitive labor. Evaluators estimated the tendency of the three types of labor in references of episodes, which illustrate various occupations such as professorship for cognitive labor, service-work for emotional labor, and construction-work for physical labor. The three labors were identified by extraction in factor analysis. On the three coordinates of labor, distances were measured between pairs of episodes. Supported with data, a dimension of cognitive labor was differentiated, constituting the three dimensions of labor as cognitive, emotional, and physical. With the independence of the three dimensions of labor, the demand and supply for each dimension are expected to be positively managed in balance for labor markets. The adoption of cognitive labor dedicates to the change of maps in labor conflicts, where traditionally problems were depicted mostly by physical and emotional labor.
\end{abstract}

Keywords: Labor Dimensions, Cognitive Labor, Emotional Labor, Physical Labor

\section{Introduction}

In daily life, labor seems to be shown in terms of physical aspects. Economic paradigms such as early liberalism, socialism, and revisionism focused on discussions of physical labor, evoking academic analyses. With the advent of postmodernism, pivoted by Derrida, the trend of the times has become multi-polarized in [1], insomuch as individual persons are interpreted in diverse social contexts in [2]. Thus, labor forces need to be analyzed as socio-psychological processes in addition to their economic material criteria.

As multi-polarized societies and high technological structures have resulted in reconsidering the roles of humans, the emergence of the service industry has been seriously attended in labor markets. In these circumstances, the individual identity as an element of an industrial organism could be complimented by the evaluation of emotional aspects. So the emotional topic has been addressed by the academic world as a major issue. Thus, a vast amount of research, estimating the values of labor by emotional criteria has been conducted.

Hochschild in [3] defined the commercialization of human feeling as the emotional labor. Since then, a great deal of discussions to refine the concept of emotional labor has evolved. The emotional labor is proposed to be differentiated from physical labor which does not composite all of business as a whole process. With respect to nonphysical labor, efforts accompanied by some kind of feelings are construed as emotional labor in [4] and [5]. Accordingly, the evaluation of emotional labor is recognizable for commercialization in [5] and [6]. This conceptualization is followed up by the need for a detailed definition of emotional labor, considering a variety of discussions on what factors and variables influence labor processes in [7] and [8]. The imposition of supervision and control on lower positioned workers by higher positioned ones, affects the expenditure of emotional labor in [9] and 
[10]. Work-places in [11] and [12], and job-statuses which require inducing or inhibiting personal feelings at service jobs in [13] and [14], teaching positions in [15] and [16], and others consume emotional labor. With the analysis of factors influencing emotion, it has been questioned how emotional burdens can be managed for the laborer's wellbeing in [17], [18], and [19]. Considering all these points, emotional labor is attested as multi-faceted and not simply contrasted to physical labor. Thus what is emergent in problems gives a way for another perspective on mental labor, which is qualified as different from emotional.

Although the discussions of emotional labor have induced the scale of some psychological values of labor, these cannot exclude that psychological processes should be analyzed in diversity and depth. What is doubted casts a question as to whether or not the emotional scale covers the whole spectrum of mental processes. Some of the analytic philosophers influenced by Schlick's 'principle of verification' in [20] belittle the concept of emotion since it is only an expression of attitude. The limitation of emotion was also raised by Moore in [21], criticizing the 'naturalistic fallacy' which regards 'good of ethic' naturally as 'pleasure in emotion'. This doctrine comments that what is told as some emotional phenomenon is owed to one of inner processes as cognitive process. So it is reasonable to posit that cognition is differentiated from emotion as far as components of the mind are concerned.

What is remarkable for cognitive factors has been discussed in dimensions of social attitude. Attitude was theorized to be divided into cognition and emotion, which are conceptualized as independent of each other in [22] and [23]. With this taxonomy, emotion is an evaluative facet of attitude, so called 'to like' or 'to dislike'. What evolves postulates that cognition is a reason of emotion in [24]. This perspective assumes that cognitive factors influence behaviors including the expressed emotion as announced by Neisser's book 'Cognitive Psychology' in [25], stating that cognition refers to the inner processes which consist of perception, memory, thought, and language. In the cognitive approaches, some of the mental processes embedded as emotional labor should be differentiated with the label of cognitive labor.

However, most researches of labor dimensions have been limited to the psychological values of emotion. So, it has been conventional to divide labors only into emotional and physical aspects without differentiating an aspect of cognitive labor from them. Without considering the short comings of having only two categories, cognitive factors have been buried under the label of emotional labor since Hochschild in [5]. Emotional labor, reviewed in most articles in [26] has been contrasted only with physical labor, and has been regarded to cover all mental requirements. This study was purposed to point out that labor processes were varied with the embedment of cognitive efforts such as those required in problem solving. For instance, in teaching jobs, which deal with problems of a student's learning in classes and troubles of adjusting to society, it is supposed that what is expended by a teacher's labor might be cognitive. So the traditional portrayal of labor disregarded a cognitive aspect which was sealed within emotional labor without differentiating one from the other.

The purpose of this experimental analysis

It is accepted that labor can be divided into physical and psychological aspects. However, a premise of a cognitive aspect should be raised, in a portion of psychological processes not only being covered by emotional aspects. In correspondence to a cognitive perspective, this research proposes a hypothesis that labor has three dimensions consisting of physical, emotional and cognitive. Based on this hypothesis, this study tried to test the distribution of these three labor dimensions, which were manipulated by three occupations such as professorship, service-work, and construction-work, where each occupation was divided into two positions leveled as typical and non-typical. The three occupations and the two positions were crossed to make six episodes for workers' job and their daily living. Each episode was judged by subjects on how much of each dimension was demanded.

It is predicted that each of the two typical levels of the three occupations have unique proportions across the three dimensions of labor; the typical position of the professorship will show a higher proportion of cognitive labor than the non-typical position, the typical level of the service work will need a greater demand for emotional labor than the non-typical level, and the typical level of the construction-work will be higher in physical labor than the non-typical level. With the experimental design this study tried primarily to differentiate cognitive labor from emotional and physical labor. Thus the research proposed to conclude the mutual independence of each aspect of labor as cognitive, emotional, and physical, and to forward a hierarchical relationship as positing the three labors at the different levels, each other.

\section{Method}

\subsection{Subject}

Seventy four students who took the course of 'the Problem of Mind', participated in this experiment as a requirement for credit for thirty minutes at Gyeongsang National University at 11:00 on May 12th 2015.

\subsection{Procedure}

Each subject responded to the questionnaire which consisted of six models in combination of three kinds of occupations and two levels of typicality on each occupation. The models were depicted as imaginary episodes of daily living, which were presented for subjects to read and respond questions followed. Each of the three occupations was thought to corresponds to one of the three aspects of labor; professorship to cognitive labor, service-work to emotional labor, and construction-work to physical labor, respectively. Each occupation was divided into two leveled 
positions, high and low, according to occupational typicality. The two levels of each occupation shared similarities in the components of daily living. However, the two positions differed in the amount of labor which was devoted with respect to each level of typicality. For example, the professor of high typicality was described like "as soon as he or she woke up, he or she browsed through the lecture notes at his or her den". On the other hand, the professor of low typicality was like "as soon as he or she woke up, he or she checked up meeting schedules". These descriptions have the same components as his or her doing on waking up, and the differentiation as reviewing lecture notes, and reminding schedules of meeting in terms of typicality of professorship.

Questions are constructed to concern two points; one is how typical the depicted model is, and the other is how much he or she consumes labors with regards to each labor dimension; cognitive, emotional, and physical labor. For additional information, the questionnaire contains three items as age, gender, and job.

Instruction was given for subjects to respond to guidelines as follows; "first read each of episodes, and then rate scores in the scale of 7 levels for each of four items under an episode". The sample of the evaluators consisted of students, participating in this research, who did not belong to any of occupations described in the episodes.

The subjects' rating scores were arranged for statistical analysis covering $t$ test, Factor analysis, and Euclidian distance in addition to mean and $S D$ processed by SPSS (v. 21.0)

\section{Results}

\subsection{The Difference Between High and Low Typicality Levels for Each Occupation}

Table 1 show how six occupational typicality levels are positioned on three dimensions of labor. It estimates some differences between the typicality levels, and among the occupations on each dimension of labors. Between the high and low typicality levels for the professorship there was a significant positive difference on the cognitive labor and a significant negative difference on the emotional labor. In other words, cognitive labor is more required for typical professor than non-typical professor, while emotional labor is more required for non-typical professor than typical professor. As for the construction-work, there was a significant difference of the physical labor between the high and low typicality levels, suggesting that the more typical construction-work is, the more physical labor is required. In contrast, for the service-work, there was a significant difference of the emotional and physical labor between the high and low typicality, suggesting that the more typical service-worker is, the more emotional and physical labor are required. In summary, the professorship was positive on demands of the cognitive labor, but negative on the emotional labor, the construction-work was consistent with the physical labor, and the service-work required a combination of the emotional and physical labor. Therefore, this suggests that labor is divided into three dimensions of physical, emotional and cognitive, which are distributed differently due to occupations and typicality levels.

Table 1. Mean, SD, and, Paired t Test between the Typical Levels of Each Occupation on Each Labor Dimension.

\begin{tabular}{|c|c|c|c|c|c|c|c|c|c|c|}
\hline & & \multicolumn{3}{|c|}{ Cognitive labor } & \multicolumn{3}{|c|}{ Emotional labor } & \multicolumn{3}{|c|}{ Physical labor } \\
\hline & & $M$ & $S D$ & $t$ & $M$ & $S D$ & $t$ & $M$ & $S D$ & $t$ \\
\hline \multirow{2}{*}{ Service } & High & 3.5946 & .7199 & \multirow{2}{*}{.673} & 4.0946 & .7479 & \multirow{2}{*}{$4.847^{* *}$} & 3.8581 & .8003 & \multirow{2}{*}{8.840 ** } \\
\hline & Low & 3.5203 & .7140 & & 3.5203 & .7694 & & 2.8851 & .7610 & \\
\hline \multirow{2}{*}{ Construction } & High & 3.3243 & 1.0930 & \multirow{2}{*}{-.666} & 3.3446 & .6617 & \multirow{2}{*}{1.267} & 3.7770 & 1.0242 & \multirow{2}{*}{$3.896 * *$} \\
\hline & Low & 3.4324 & .9002 & & 3.2027 & .7352 & & 3.1689 & .9661 & \\
\hline \multirow{2}{*}{ Professor } & High & 3.9459 & .8781 & \multirow{2}{*}{$4.500^{* *}$} & 3.2432 & .7905 & \multirow{2}{*}{$-3.925 * *$} & 2.9730 & .9286 & \multirow{2}{*}{-.986} \\
\hline & Low & 3.3378 & .8071 & & 3.7568 & .7992 & & 3.1149 & .8896 & \\
\hline
\end{tabular}

\subsection{The Differentiation of Three Labor Dimensions-Cognitive, Emotional, and Physical}

Concerning the analysis above, labor is differentiated into physical, emotional and cognitive dimension, which varies according to occupational typicality levels. So whether or not labor dimensions are independent of each other comes into question. To test the correlation structure of labor dimensions, factor analysis was processed. To find the correspondence between a typical occupation and its dimension of labor, the correlation matrix of three typical occupations, and three dimensions of labor was analyzed for factor extraction. The result of the factor analysis is shown in Table 2 (KMO, Bartlett test - Varimax rotation). Analyzing the matrix, since the three factors were extracted with the accumulative variance close to $100 \%$, it was concluded that no other factors were to be joined. Ranking proportions of distribution, the first factor corresponds to the physical labor of the typical construction-work, the second relates to the emotional labor of the typical service-work, and the third sets for the cognitive labor of the typical professorship. Therefore this suggests that labor can be differentiated into physical, emotional, and cognitive dimension. 
Table 2. Factor Analysis with the Matrix of the Typical Professorship-Cognitive Labor, the Typical Service-Emotional Labor, and the Typical ConstructionPhysical Labor.

\begin{tabular}{|c|c|c|c|c|c|c|c|c|c|}
\hline \multirow{2}{*}{ Component } & \multicolumn{3}{|l|}{ Initial } & \multicolumn{2}{|c|}{ Extracted } & & \multicolumn{3}{|c|}{ Rotated } \\
\hline & Sum & $\%$ Var & $\%$ Cum & Sum & $\%$ Var & $\%$ Cum & Sum & $\%$ Var & $\%$ Cum \\
\hline 1 & 1.721 & 57.364 & 57.364 & 1.721 & 57.364 & 57.364 & 1.006 & 33.522 & 33.522 \\
\hline 2 & .782 & 26.074 & 83.438 & .782 & 26.074 & 83.438 & 1.005 & 33.511 & 67.033 \\
\hline 3 & .497 & 16.562 & 100.000 & .497 & 16.562 & 100.000 & .989 & 32.967 & 100.000 \\
\hline \multicolumn{10}{|c|}{ Rotated matrix } \\
\hline & & \multicolumn{2}{|c|}{ Component } & \multicolumn{6}{|c|}{ Commonality } \\
\hline & & \multicolumn{2}{|c|}{1} & 2 & 3 & \multicolumn{2}{|c|}{ Initial } & \multicolumn{2}{|c|}{ Extracted } \\
\hline \multicolumn{2}{|c|}{ Physical-Typical Construction } & \multicolumn{2}{|c|}{.978} & .091 & .188 & \multicolumn{2}{|c|}{1.000} & \multicolumn{2}{|c|}{1.000} \\
\hline \multicolumn{2}{|c|}{ Emotional-Typical Service } & \multicolumn{2}{|c|}{.092} & .971 & .219 & \multicolumn{2}{|c|}{1.000} & \multicolumn{2}{|c|}{1.000} \\
\hline \multicolumn{2}{|c|}{ Cognitive-Typical Professor } & \multicolumn{2}{|c|}{.202} & .231 & .952 & \multicolumn{2}{|c|}{1.000} & \multicolumn{2}{|c|}{1.000} \\
\hline
\end{tabular}

\subsection{The Distances of Occupational Typicality Levels on the Three Labor Dimensions}

As shown above, labor that is required differently for two occupational typicality levels is differentiated into physical, emotional, and cognitive dimension. Thus, the points of each occupational typicality level were drawn on the three dimensions, and the distances between pairs of them were calculated. The means of each occupational typicality level on the cognitive, emotional, and physical labor are coordinated, as follows in Table 1. With the coordinates of the cognitive, emotional, and physical dimension, the distances between each pair in matrix of the six by six occupational typicality levels are shown in Table 3. With regards to the distances, one between the typical and nontypical levels on the same occupations was short only for the construction-work. Besides this, the other distances between the typicality levels within each occupation were rather further apart than some pairs between the occupations. Analyzing this, the construction-work, disregarding the typicality levels, requires mainly the physical labor, but the typicality levels of the other two occupations are complicated to share the cognitive and emotional labor. The typical service-work that requires the high emotional and high physical labor is near the typical construction-work and the non-typical professorship. The non-typical service-work with the low physical labor is near the non-typical constructionwork and the professorship. Also, the non-typical construction-work which requires labors of all three dimensions is near the non-typical service-work and the nontypical professorship. The professorship is near the nontypical service-work and the non-typical construction-work in regards to the emotional labor. What these results suggest is distances are not related by superficial divisions of occupation, but are varied due to labor dimensions analyzed in depth.

Table 3. Distances Calculated between All Pairs among the Occupation-Typical Levels.

\begin{tabular}{|c|c|c|c|c|c|c|}
\hline & \multicolumn{6}{|c|}{ Squared Euclidean Distance } \\
\hline & \multicolumn{2}{|c|}{ Service } & \multicolumn{2}{|l|}{ Construction } & \multicolumn{2}{|c|}{ Professorship } \\
\hline & -High & -Low & -High & -Low & -High & -Low \\
\hline \multicolumn{7}{|c|}{ Service } \\
\hline -High & .000 & 1.282 & .642 & 1.297 & 1.632 & .732 \\
\hline -Low & 1.282 & .000 & .865 & .189 & .266 & .142 \\
\hline \multicolumn{7}{|c|}{ Construction } \\
\hline -High & .642 & .865 & .000 & .402 & 1.043 & .608 \\
\hline -Low & 1.297 & .189 & .402 & .000 & .304 & .319 \\
\hline \multicolumn{7}{|c|}{ Professorship } \\
\hline -High & 1.632 & .266 & 1.043 & .304 & .000 & .654 \\
\hline -Low & .732 & .142 & .608 & .319 & .654 & .000 \\
\hline
\end{tabular}

\section{Discussions}

This study tried to supplement labor dimensions in correspondence to postmodern discussions that paid attention to a subjective world, renewing perspectives that human labor is not limited to objective physical values. Thus in terms of psychological value, the concept of emotional labor is appreciated for a new theory. While a lot of preceding researches has been conducted with diverse contexts, there remains a conceptual problem that all of psychological labor is generally buried under the umbrella of only an emotional dimension. Following the initial cognitive approaches and the present cognitive scientists, the purpose of this study was to testify a scheme of three dimensions, composed of cognitive, emotional, and physical labor, differentiating the cognitive one from the other two.

The results of this study supported the hypothesis proposing the differentiation of cognitive, emotional and physical labor dimension, tested by Factor-Analysis and other statistics. The two levels of typicality were differentiated as expected on occupations of professorship, 
service-work, and construction-work. The conditions of the three occupations divided into two typical levels were differentiated on the three labor dimensions. In detail, the two typical levels of professorship had a positive difference for cognitive labor, and a negative difference for emotional labor. The two typical levels of service-work were different on emotional and physical labor, while the two typical levels of construction-work differed only on physical labor. Hereby the three labor dimensions are differentiated, and the labors that each occupational typical level required were identified. Related to this, the three coordinates, along which the six conditions of occupational typical levels were settled in dimensions, and also the distances paired with the six conditions were confirmed. In detail, there are some pairs of typicality levels that are nearer between occupations than within each occupation, suggesting that the distances are varied owing more to the labor dimensions than to occupation divisions.

Summarizing the results, labor dimensions can be extended beyond physical and emotional labor. It is commented with confidence that emotional labor does not substitute the whole of psychological processes, which was once proposed to overcome the limitation of physical labor. This analysis supports the proposition to differentiate a new dimension of cognitive labor, constituting a total of three dimensions of labor.

\section{Conclusions}

This study is significantly evaluated in that it independently differentiates cognitive labor. Traditionally, the concept of labor has been limited to physical labor, and then developed to consider psychological labor, with a sole focus on emotional aspect. Previous studies on emotional labor have either estimated the degree of fatigue after labor without regards to the quantity of physical work in [26] and [3], or measured the degree of psychological consumption when the reward for labor fell short of expectations in [6] and [12]. With these scales, the assumed emotional labor cannot cover the whole spectrum of psychological labor, and as this study pointed out, cognitive labor needs to be institutionalized.

Since emotion is to evaluate the consciousness and behavior of individuals expressing their attitudes, what works for causative processes is questioned. This underlying process is posited as cognition in a cognitive paradigm. Such a cognitive approach was initialized once by J Piaget in [27], who propounded that the concrete or formal operation, known as cognitive competence develops to govern the coordination of the mind and body. Since then, many cognitive psychologists have insisted that overall consciousness and behavior, including emotion can be explained with the activation of cognitive networks in [28] and [29]. So, at the foundation of overall consciousness, precede cognitive processes. In this perspective, emotion as a secondary process cannot cover the whole range of psychological processes that occur, but is one of apparent consciousness and behavior. On aspects of labor, cognitive efforts are initialized, and then the concrete labors of emotional motives and physical activities follow. This comment surpasses even the theory of three dimensions of cognitive, emotional, and physical labor, renewing a proposition that cognitive effort lies at a basic level, on which emotional motives ride, and go ahead onto physical activity. What is implied now sets a hierarchical structure where the levels of labor circulate, positing cognitive effort on base.

One of suggestions which would be emerged from this study for the three dimensions of labor concerns what is issued in social problems of labor. Only regarding the physical labor, the labor conflicts were dealt in the traditional economic ideologies. By the refinement of emotional labor, some changes of the labor troubles are foreseen in [30]. Moreover it is drawn that the map of labor problems is complicated by the inclusion of cognitive labor to social system.

\section{Acknowledgements}

Sih Lee (the first author) initiated the idea of this research to propose the experimental design, accomplished experimental processes, and drafted the overview of this article. Junghye Jeong (the second author) advised the experimental processes, and processed statistics for this research data. Yang Lee (the correspondent author) revised the idea and the experimental design, and discussed the theoretical issues with revision of this article. For compliance with ethical standards, no one of authors has supports of any research fund for this study, and the authors declare that they have no conflict of interest. This study complies in rules by Gyeongsang National University Institute Review Board for Human participants.

\section{References}

[1] V. W. Cisney. Derrida's voice and phenomenon: An Edinburgh philosophical guide. Edinburgh: Edinburgh University Press, 2014.

[2] E. Durkheim. The division of labor in society. (W. D. Halls, Trans.) New York, NY: Free Press, 2014.

[3] A. R. Hochschild. Emotion work, feeling rules, and social structure. American Journal of Sociology, 85, 1979: 551-575.

[4] C. M. Brotheridge, \& A. A. Grandey. Emotional labor and burnout: Comparing two perspectives of people work. Journal of Vocational Behavior, 60, 2002: 17-39.

[5] A. R. Hochschild. The managed heart. Berkeley, CA: University of California Press, 1983.

[6] S. M. Kruml, \& D. Geddes. Exploring the dimensions of emotional labor: The heart of Hochschild's work. Management Communication Quarterly, 14, 2000: 8-49.

[7] A. A. Grandey, \& R. C. Melloy. The state of the heart: Emotional labor as emotion regulation reviewed and revised. Journal of Occupational Health Psychology, 22, 2017: 407-422. 
[8] B. Mesquita, \& E. Delvaux. Emotional labor in the $21 \mathrm{st}$ century: Diverse perspectives on emotion regulation at work. Abingdon: Routledge Psychology Press, 2013.

[9] R. J. Erickson, \& C. Ritter. Emotional labor, burnout, and inauthenticity: Does gender matter? Social Psychology Quarterly, 64, 2001: 146-163.

[10] H. Kim, W. Hur, T. Moon, \& J. Jun. Is all support equal? The moderating effects of supervisor, coworker, and organizational support on the link between emotional labor and job performance. Business Research Quarterly, 20, 2017: 124-136.

[11] A. A. Grandey. Emotional regulation in the workplace: A new way to conceptualize emotional labor. Journal of Occupational Health Psychology, 5, 2000: 95-110.

[12] J. Schaubroeck, \& J. R. Jones. Antecedents of workplace emotional labor dimensions and moderators of their effects on physical symptoms. Journal of Organizational Behavior, 21, 2000: 163-183.

[13] B. E. Ashforth, \& R. H. Humphrey. Emotional labor in service roles: The influence of identity. Academy of Management Review, 18, 1993: 88-115.

[14] H. J. Kim. Hotel service providers' emotional labor: The antecedents and effects on burnout. International Journal of Hospitality Management, 27, 2008: 151-161.

[15] A. Ghanizadeh, \& N. Royaei. Emotional facet of language teaching: Emotion regulation and emotional labor strategies as predictors of teacher burnout. International Journal of Pedagogies and Learning, 10, 2015: 139-150.

[16] X. Qi, S. Ji, J. Zhang, W. Lu, J. K. Sluiter, \& H. Deng. Correlation of emotional labor and cortisol concentration in hair among female kindergarten teachers. International Archives of Occupational and Environmental Health, 90, 2017: $117-122$.

[17] S. H. Mastracci, M. E. Guy, \& M. A. Newman. Emotional labor and crisis response: Working on the razor's edge. Abingdon: Routledge Taylor \& Francis Group, 2014.
[18] E. Wong, F. Tschan, \& N. K. Semmer. Effort in emotion work and well-being: The role of goal attainment. Emotion, 17, 2017: 67-77.

[19] D. Zapf. Emotion work and psychological well-being: A review of the literature and some conceptual considerations. Human Resource Management Review, 12, 2002: 237-268.

[20] W. Leinfellner. A reconstruction of Schlick's psychosociological ethics. Syntheses, 64, 1985: 317-349.

[21] G. E. Moore. Principia ethica. Cambridge: Cambridge University Press, 1903.

[22] C. E. Izard, J. Kagan, \& R. B. Zajonc. Emotions, cognition, and behavior. Cambridge: Cambridge University Press, 1984.

[23] R. V. Wagner, \& J. J. Sherwood. The study of attitude change. Monterey, CA: Books Cole Publishing Company, 1969.

[24] V. Hamilton, G. H. Bower, \& N. H. Frijda. Cognitive perspectives on emotional motivation. Norwell, MA: Kluwer Academic Publisher, 1988.

[25] U. Neisser. Cognitive psychology. New York, NY: Meredith, 1967.

[26] C. E. J. Härtel, N. M. Ashkanasy, \& W. J. Zerbe. What have we learned? Ten years on. Bingley: Emerald Group Publishing Limited, 2011.

[27] H. Ginsberg, \& S. Opper. Piaget's theory of intellectual development. Englewood Cliffs, NJ: Printice-Hall, 1979.

[28] A. M. Collins, \& R. M. Quillian. Retrieval time from semantic memory. Journal of Verbal Learning and Verbal Behavior, 8, 1969: 240-247.

[29] D. E. Rumelhart, \& J. L. McClelland. Parallel distributed processing. Cambridge, MA: Massachusetts Institute of Technology Press, 1986.

[30] C. M. Barnes, K. Jiang, \& D. P. Lepak. Sabotaging the benefits of our own human capital: Work unit characteristics and sleep. Journal of Applied Psychology, 101, 2016: 209-221. 